\title{
Psicooncología
}

ISSN: 1696-7240

http://dx.doi.org/10.5209/PSIC.63648

\section{Escala de Afrontamiento al Cáncer en pacientes oncológicos en población mexicana}

\author{
Oscar Galindo Vázquez ${ }^{1 *}$
}

Recibido el 7 de noviembre de 2018 / Aceptado el 7 de marzo de 2019

Resumen. Introducción: Los estilos de afrontamiento son variables psicológicas que pueden predecir la adaptación psicológica y en sentido contrario una mayor disfunción psicosocial en pacientes con cáncer. Se han descrito dos dimensiones: centrados en el problema o activos positivos, caracterizados por la búsqueda de información, planificación y resolución de problemas; y los dirigidos a la emoción o evitativos caracterizados por el escape, distanciamiento o desesperanza. Objetivo: Determinar las propiedades psicométricas de la Escala de Afrontamiento del Cáncer en una muestra de pacientes con cáncer de la población mexicana. Método: Participaron 317 pacientes del Instituto Nacional del Cáncer, de los cuales 148 eran mujeres $(46,7 \%)$ y 169 hombres $(53,4 \%)$, con una edad media de 46,2 años. Los participantes completaron al mismo tiempo el Mental Adjustment to Cancer (MAC), el HADS y el Termometro del Distress. Resultados: Mediante el análisis factorial se identificaron cinco factores que explicaron el 53,32\% de la varianza, con 22 reactivos. La consistencia interna de la escala global fue satisfactoria $(0,81)$. La validez concurrente mostró asociaciones significativas (Pearson $\mathrm{r}=-0,120$ $-0,586, p<0,05)$. Discusión y conclusión: La Escala de Afrontamiento del Cáncer derivada del Mental Adjustment to Cancer tiene propiedades psicométricas adecuadas. Es una herramienta útil para fines de investigación y atención en salud mental durante el tratamiento oncológico.

Palabras clave: cáncer, afrontamiento, escala, pacientes, población mexicana.

\section{[en] Scale of coping with cancer in cancer patients in the Mexican population}

\begin{abstract}
Introduction: Coping styles can predict psychological adaptation and in the opposite direction a greater psychosocial dysfunction in patients with cancer. Two dimensions have been described: focused on the problem or positive assets, characterized by the search for information, planning and problem solving; and those directed at emotion or avoidant characterized by escape, distancing or despair have been consistently described in the literature. Objective: Determine the psychometric properties of the Mental Adjustment to Cancer (MAC) in a sample of patients with cancer from the Mexican population. Method: Three hundred seventeen patients from the National Cancer Institute participated, of which 148 were women (46.7\%) and 169 men (53.4\%), with a mean age of 46.2 years. Completed concurrently the Mental Adjustment to Cancer, HADs and Distress Thermometer. Results: A factor analysis show to five factors explained $53.32 \%$ of the variance, with 22 items loading on factors in a way similar to the original version. The internal consistency of the overall scale was satisfactory $(0.81)$. The concurrent validity assessed by way of correlations with concurrent measures showed significant associations (Pearson $\mathrm{r}=-0.120-0.586, \mathrm{p}<0.05$ ). Discussion and conclusion: The Cancer Coping Scale extracted from MAC has adequate construct validity,

1 Oscar Galindo Vázquez. Servicio de Psico-oncología, Instituto Nacional de Cancerología INCan. México

* Correspondencia: Dr. Oscar Galindo Vázquez. Servicio de Psico-oncología, Instituto Nacional de Cancerología. Av. San Fernando 22. Sección XVI, Tlalpan, 14080, Ciudad de México. E-mail: psigalindo@yahoo.com.mx
\end{abstract}


internal consistency and concurrent validity for its use in cancer patients from the Mexican population. The relevance of these results is a cost effective tool to provide timely mental health care early in oncological treatment for those in need.

Keywords: cancer, coping, scale, patients, mexican population.

Sumario. 1. Introducción 2. Método 3. Análisis 4. Resultados 5. Discusión 6. Referencias bibliográficas.

Cómo citar: Galindo Vázquez O. Escala de Afrontamiento al Cáncer en pacientes oncológicos en población mexicana. Psicooncología 2019; 16(1): 61-72. doi: 10.5209/PSIC.63648.

\section{Introducción}

En las últimas décadas ha habido un interés creciente por investigar las distintas demandas a las que se enfrenta el paciente con cáncer y sobre todo, por fomentar estrategias de afrontamiento que le permitan hacer frente a dichas demandas ${ }^{(1)}$. El diagnóstico y tratamiento del cáncer implican un proceso de adaptación a múltiples amenazas y nuevas experiencias. El cómo las características individuales evalúan y responden a estas amenazas y experiencias se le conoce como estilo de afrontamiento $^{(2)}$.

De a acuerdo a $\mathrm{Nezu}^{(3)}$ el cáncer y su tratamiento representa para los pacientes eventos estresantes que pueden generar repercusiones psicológicas. Frente a estas situaciones las personas plantean diferentes estilos de afrontamiento, definidos como aquéllos esfuerzos cognitivos y conductuales constantemente cambiantes que se desarrollan para manejar las demandas específicas externas y/o internas que son evaluadas como excedentes o desbordantes de los recursos del individuo ${ }^{(4)}$.

Se han identificado tres constructos utilizados comúnmente para abordar el afrontamiento en población oncológica: a) los estilos de afrontamiento, b) las estrategias de afrontamiento, y c) el ajuste mental al cáncer ${ }^{(5)}$. Los estilos de afrontamiento que son entendidos como formas estables y consistentes de afrontar el estrés. Las estrategias de afrontamiento planteadas por Tobin, Holroyd, Reynolds y $\mathrm{Kigal}^{(6)}$. Dichas estrategias se definen como acciones específicas que pueden variar dependiendo del contexto de la situación estresante. Los autores citados extrajeron niveles generales de análisis, los cuales permiten identificar dos tipos de estrategias: a) las centradas en la aproximación, que representan esfuerzos cognitivos y conductuales para encarar y controlar directamente el evento estresante, y b) las centradas en la evitación, las cuales implican una reducción de la tensión mediante el escape del estresor ${ }^{(5)}$.

Por otro lado se ha planteado el modelo de Ajuste Mental al Cáncer de Watson ${ }^{(7,8)}$ el cual ha sido ampliamente utilizado para evaluar las respuestas cognitivas y conductuales del paciente ante al diagnóstico de cáncer ${ }^{(9)}$. Este modelo está centrado en el significado personal que tiene el cáncer para el individuo y las estrategias de afrontamiento que se ponen en marcha para lograr su adaptación. Estos autores proponen un esquema de supervivencia o una triada de evaluación cognitiva, para la cual se toman en cuenta tres aspectos: (a) la percepción del diagnóstico, que responde a la pregunta "¿qué significado tiene para usted esta enfermedad?”; (b) la sensación de control sobre la situación, responde a las preguntas "¿qué puede 
hacerse frente a la enfermedad?” y “ ¿se puede controlar?” y (c) la visión acerca del pronóstico, que contesta a la pregunta "¿cuál es el pronóstico de la enfermedad y si este pronóstico es confiable?". Independientemente de la situación real de pronóstico favorable o no del paciente, esta visión se relaciona con los dos aspectos mencionados anteriormente.

Estos aspectos definen cinco tipos de afrontamiento que presenta al individuo, que permiten sobrellevar el cáncer y que tendrán su consecuente respuesta emocional ${ }^{(8)}$. Así los tipos de ajuste y la respuesta emocional que tiene el individuo frente a su estado de salud dependen de la evaluación que el sujeto hace de la enfermedad. Los cinco estilos son: Espíritu de Lucha, Evitación/Negación, Fatalismo/Aceptación Estoica, Desamparo/Desesperanza y Preocupación Ansiosa. En este contexto la Escala de Ajuste Mental al Cáncer (MAC) es un instrumento ampliamente utilizado que mide estos cinco tipos de afrontamiento en un medio hospitalario y ambulatorio, que se asocian con diferentes formas de adaptación ante el cáncer ${ }^{(10)}$.

El MAC en su versión extensa ${ }^{(10)}$ y breve ${ }^{(11)}$ han sido adaptados y validados en diversas poblaciones como pacientes en tratamiento curativo y paliativo; así como en diversos países europeos (Francia. Italia y Grecia) ${ }^{(12-14)}$, iberoamericanos (España y Colombia) ${ }^{(15,16)}$ y asiáticos (China, Japón y Korea) ${ }^{(17-19)}$, en todos ellos ha mostrado una adecuada estructura factorial en la discriminación de los diferentes tipos de afrontamiento.

En diversas poblaciones oncológicas ambas versiones ${ }^{(10,11)}$ han demostrado ser un instrumento con una adecuada consistencia interna (alfas entre 0,60 y 0,93 ), alta confiabilidad test retest $(r=0,75)$, una adecuada validez convergente $(0,75)$, rangos de sensibilidad entre 0,70 y 0,83 y especificidad de 0,75 a 0,85 para identificar los diferentes estilos de afrontamiento, así como una estructura factorial congruente/ similar con lo reportado por los autores originales ${ }^{(12-21)}$. Por lo que el objetivo del presente estudio es determinar las propiedades psicométricas de la Escala de Afrontamiento al Cáncer derivada del MAC traducido de la versión extensa para población oncológica mexicana.

\section{Método}

Se incluyeron 317 pacientes oncológicos de ambos sexos con un promedio de 46,28 años de edad (Ver la tabla 1 para una descripción de la muestra). La muestra de participantes se obtuvo durante las consultas de los servicios de oncología médica, cirugía, radioterapia y psico-oncología.

\section{Procedimiento}

Alos participantes se les invitó explicándoles el objetivo del estudio, riesgo ybeneficios; después de la comprensión del mismo y si aceptaban participar, se les solicitó que firmaran el documento de Consentimiento Informado. La cumplimentación de los instrumentos se llevó a cabo en aproximadamente 20 minutos. La aplicación de los instrumentos las realizó el equipo del Servicio de Psicooncología del INCan. Esta investigación fue aprobada por el comité de investigación y el comité de ética en investigación del INCan. 
Tabla 1. Descripción de la muestra

\begin{tabular}{|c|c|c|c|c|c|}
\hline & f & $\%$ & & f & $\%$ \\
\hline $\mathrm{N}$ & 317 & 100 & & & \\
\hline \multirow[t]{2}{*}{ Edad (rango) 17-71 años } & $x=46,28$ & & Diagnóstico & & \\
\hline & & & Cáncer de mama & 89 & 28,1 \\
\hline Genero & & & Cabeza y cuello & 44 & 13,9 \\
\hline Masculino & 148 & 46,7 & Urológico & 63 & 19,9 \\
\hline \multirow[t]{3}{*}{ Femenino } & 169 & 53,3 & Gástricos & 15 & 4,7 \\
\hline & & & Ginecológicos & 26 & 8,2 \\
\hline & & & Neumología & 24 & 7,6 \\
\hline Escolaridad & & & Otros & 56 & 17,6 \\
\hline Ninguna & 10 & 3,2 & & & \\
\hline Primaria & 56 & 17,7 & Estadio & & \\
\hline Secundaria & 97 & 30,6 & I & 18 & 5,7 \\
\hline Bachillerato & 76 & 24,0 & II & 98 & 30,9 \\
\hline Licenciatura & 67 & 21,0 & III & 127 & 40,1 \\
\hline \multirow[t]{2}{*}{ Posgrado } & 11 & 3,5 & IV & 74 & 23,3 \\
\hline & & & Tratamiento & & \\
\hline Estado civil & & & Quimioterapia & 193 & 60,9 \\
\hline Soltero(a) & 87 & 27,4 & Cirugía & 31 & 9,8 \\
\hline Casado(a)/Juntado & 168 & 53,0 & Radio/Quimio & 54 & 17,0 \\
\hline Divorciado(a) & 49 & 15,5 & Radioterapia & 17 & 5,4 \\
\hline \multirow[t]{2}{*}{ Viudo(a) } & 13 & 4,1 & Otros & 22 & 6,9 \\
\hline & & & Enfermedad comorbida & & \\
\hline Paternidad & & & $\mathrm{Si}$ & 74 & 23,3 \\
\hline $\mathrm{Si}$ & 226 & 71,3 & No & 243 & 76,7 \\
\hline \multirow[t]{3}{*}{ No } & 91 & 28,7 & & & \\
\hline & & & Índice Karnofsky & & \\
\hline & & & 60 & 2 & 0,6 \\
\hline 0 & 162 & 51,1 & 70 & 14 & 4,4 \\
\hline 0 & 45 & 14,2 & 80 & 69 & 21,8 \\
\hline 1 & 71 & 22,4 & 90 & 156 & 49,2 \\
\hline 2 & 35 & 11,0 & 100 & 78 & 24,6 \\
\hline 3 & 2 & 0,6 & & & \\
\hline 4 & 1 & 0,3 & & & \\
\hline
\end{tabular}

Los participantes fueron seleccionados a partir de los criterios de inclusión: (a) pacientes en tratamiento oncológico (cualquier tipo de tratamiento), (b) cualquier tipo de diagnóstico y estadio oncológico, (c) saber leer y escribir y (d) Índice Karnofsky igual o mayor a 60. Los criterios de exclusión fueron: (a) pacientes con cuadros psiquiátricos severos y/o adicción a alguna sustancia psicoactiva, (b) en crisis psicológica, (c) con disnea, (d) deficiencias cognitivas y/o (e) problemas auditivos y/o visuales severos que impidieran contestar los instrumentos. 


\section{Instrumentos}

Se administraron los siguientes instrumentos acompañados con una cédula de identificación de datos sociodemográficos (edad, género, escolaridad, estado civil, paternidad) y clínicos (comorbilidad, índice Karnofsky, diagnóstico, estadio y tratamiento).

\section{Escala de Ajuste Mental al Cáncer - MAC -}

Se realizó la traducción y re-traducción de la versión extensa del MAC al castellano por dos traductores expertos, un nuicio por expertos y una prueba piloto con el objetivo de evaluar la redacción, expresiones confusas y dificultad, posteriormente realizar los cambios pertinentes. Quedo un instrumento compuesto por 40 ítems que miden cinco tipos de afrontamiento en un medio hospitalario y ambulatorio: (a) espíritu de lucha, (b) desesperanza/desamparo, (c) preocupación ansiosa, (d) fatalismo/aceptación estoica y (d) negación; todos ellos que asocian con diferentes formas de adaptación ante el cáncer. En diversas poblaciones oncológicas este instrumento ha mostrado una adecuada consistencia interna (alfas entre 0,60 y 0,93 ), alta confiabilidad test retest $(\mathrm{r}=0,75)$ y una adecuada validez convergente 0,75 y rangos de sensibilidad entre 0,70 y 0,83 , especificidad de 0,75 a 0,85 para identificar los diferentes estilos de afrontamiento ${ }^{(12-21)}$.

\section{Escala Hospitalaria de Ansiedad y Depresión - HADS -}

La Escala Hospitalaria de Ansiedad y Depresión (HADS) ${ }^{(22)}$ es una escala autoaplicada conformada por 14 ítems que permite detectar síntomas de ansiedad y depresión en un medio hospitalario no psiquiátrico o en medios de atención primaria. Esta escala consta de dos subescalas de 7 ítems intercalados. La subescala de depresión está centrada en el concepto de anhedonia como síntoma nuclear de este cuadro clínico. Dicho instrumento ha sido validado en población mexicana ${ }^{(23)}$ la cual mostró una estructura factorial con 12 ítems y una adecuada consistencia interna de la escala global $(\alpha=0,82)$. Las alfas de Cronbach de cada subescala son de 0,80 y 0,79 que explican el $48,04 \%$ de la varianza. En relación a la validez concurrente entre el HADS, Inventario de Depresión de Beck, Inventario de Ansiedad de Beck y Termómetro de Distrés se obtuvieron correlaciones positivas y estadísticamente significativas ( $\mathrm{r}$ de Pearson de 0,51 a $0,71, \mathrm{p}<0,05$ ).

El Termómetro de Distrés ${ }^{(24)}$ validada para población mexicana ${ }^{(25)}$ consta de dos partes. La primera es una escala análoga visual, ubicada en forma vertical y bajo la apariencia de un termómetro. En la segunda parte se solicita que de una lista de 35 problemas físicos y emocionales el sujeto señale la existencia o no de los mismos. Las propiedades del termómetro de Distrés en población mexicana muestran una confiabilidad por consistencia interna adecuada: $\alpha=0,87$. La validación convergente a través del Análisis ROC mostró un área bajo la curva de 0,63, sensibilidad de 93\%, especificidad de $76 \%$, valor predictivo positivo de $82 \%$, valor predictivo negativo de $90 \%$, con un punto de corte de cuatro o más. 


\section{Análisis}

Para el análisis de los datos se usó el paquete estadístico SPSS versión 14.0 para Windows. Se obtuvo los estadísticos de frecuencias de cada reactivo para conocer su distribución en las opciones de respuestas; posteriormente se determinaron los grupos extremos (cuartil 25 y 75) basados en la calificación obtenida por los sujetos participantes y con cada reactivo se llevó a cabo una prueba t de Student para muestras independientes para identificar la propiedad de discriminación.

Posteriormente se realizaron análisis de tablas cruzadas para conocer la correlación entre ítems. Se determinó la estructura factorial por medio de un análisis factorial de componentes principales con rotación varimax. Los criterios utilizados para el análisis factorial fueron: (a) cargas factoriales $\geq 0,40$ y (b) ítems que cargaran sólo en un factor. La relación entre la versión derivada del MAC, HADS y termómetro de distrés se analizó mediante el coeficiente de correlación de Pearson, con un nivel de confianza del $95 \%$.

\section{Resultados}

\section{Estructura factorial}

Se obtuvo en la prueba de esfericidad de Bartlett $(p=0,001)$ y un índice de adecuación muestral $(\mathrm{KMO}=0,879)$ que determinó la suficiencia de la muestra para la estructura de los componentes finales. El análisis factorial (método de componentes principales y rotación varimax) con 40 reactivos arrojó un modelo con cinco factores con autovalores arriba de 1,00. Los reactivos 1, 5, 8, 10, 12, 15, 16, 19, 21, 23, 24, 25, $26,27,28,29,33$ y 35 fueron eliminados de acuerdo con los criterios para el análisis factorial antes mencionados ver tabla 2.

\section{Consistencia interna}

La consistencia interna de la escala global mostró un índice adecuado $(\alpha=0,89)$. Se identificó una estructura de cinco factores: espíritu de lucha, preocupación ansiosa, desamparo/desesperanza, actitud positiva y evitación cognitiva que explican el $53,32 \%$ de la varianza. En términos generales, la consistencia puede considerarse adecuada, ver tabla 2 .

\section{Validez externa}

En la tabla 6 muestra el resumen de las asociaciones entre el MAC-M, sus subescalas, la HADS-M y Termómetro de Distrés. Como se puede observar las correlaciones fueron positivas o negativas dependiendo de la relación teórica entre subescalas, de magnitud baja a media y estadísticamente significativas $(r$ de Pearson de $-0,367$ a $0,586, p<0,05, p<0,01)$ ver tabla 3 . 
Tabla 2. Análisis factorial, consistencia interna y varianza explicada

\begin{tabular}{lc}
\hline $\begin{array}{l}\text { Escala Global } \alpha=.81 \\
\text { Varianza Explicada }=53.32 \%\end{array}$ & $\begin{array}{c}\text { Peso } \\
\text { factorial }\end{array}$ \\
\hline $\begin{array}{l}\text { Factor I, Espíritu de lucha } \\
\text { 11. Desde que me diagnosticaron el cáncer } \\
\quad \text { valoro mucho más mi vida y trato de sacar }\end{array}$ &, 693 \\
$\quad$ el mayor provecho de ella. & \\
20. Estoy decidido(a) a enfrentar de todo. &, 798 \\
31. Trato de tener una actitud positiva. &, 644 \\
34. Veo mi enfermedad como un desafío. &, 494 \\
39. Me concentro en los aspectos buenos que &, 684 \\
Tengo. &, 754 \\
40. Intento luchar contra la enfermedad.
\end{tabular}

Factor II. Preocupación ansiosa

3. Mis problemas de salud me impiden hacer planes para el futuro.

14. Me preocupa que el cáncer vuelva a $\quad$,681 aparecer o empeore.

22. Siento mucha ansiedad a causa de la $\quad, 803$ enfermedad.

36. No sé lo que debo de hacer. $\quad$,452

37. Estoy muy enfadado(a) por lo que me está $\quad, 737$ ocurriendo.

Factor III. Desamparo/Desesperanza

2. Siento que no puedo hacer nada para

alegrarme o sentirme mejor

7. Creo que nada de lo que pueda hacer $\quad, 709$ cambiaran las cosas

9. Pienso que mi vida no tiene sentido. $\quad, 639$

17. Creo que no hay nada que yo pueda hacer para $\quad$,718 ayudarme.

30. Creo que no puedo controlar lo que me acurra. $\quad$,568

\section{Factor IV. Actitud positiva}

4. Creo que una actitud positiva será $\quad, 671$

$$
5,36 \% \quad \alpha=, 692
$$
beneficioso(a) para mi salud.

6. No tengo ninguna duda de que mejoraré. $\quad$,768

13. Tengo planes para el futuro (por ejemplo $\quad, 606$ tener vacaciones, trabajo, cambios importantes).

\section{Factor V. Evitación cognitiva}

$$
4,69 \% \quad \alpha=, 449
$$

18. Intento seguir viviendo como siempre lo he ,613 hecho.

32. Estoy tan ocupado(a) que no tengo tiempo $\quad, 642$ para pensar en el cáncer.

38. En realidad yo no creo que tenga cáncer. 
Tabla 3. Correlaciones entre la escala de Afrontamiento al Cáncer, HADS y Termómetro de Distrés

\begin{tabular}{lccccccc}
\hline & $\mathbf{1}$ & $\mathbf{2}$ & $\mathbf{3}$ & $\mathbf{4}$ & $\mathbf{5}$ & $\mathbf{6}$ & $\mathbf{7}$ \\
\hline 1. Espíritu de lucha & 1 & & & & & & \\
2. Preocupación ansiosa & $-0,143^{* *}$ & & & & & & \\
3. Desamparo/Desesperanza & $-0,304^{*}$ & $0,458^{* *}$ & & & & & \\
4. Actitud positiva & $0,534^{* *}$ & $-0,210^{* *}$ & $-0,399^{* *}$ & & & & \\
5. Evitación cognitiva & $0,334^{* *}$ & $-0,099$ & $-0,074$ & $0,252^{* *}$ & & & \\
6. HADS Ansiedad & $-0,209^{* *}$ & $0,586^{* *}$ & $0,302^{* *}$ & $-0,195^{* *}$ & $-0,205^{* *}$ & & \\
7. HADS Depresión & $-0,330^{* *}$ & $0,421^{* *}$ & $0,374^{* *}$ & $-0,367^{* *}$ & $-0,197^{* *}$ & $0,619^{* *}$ & \\
8. Distrés & $-0,011$ & $0,398^{* *}$ & $0,157^{* *}$ & $-0,120^{*}$ & $-0,138^{* *}$ & $0,569^{* *}$ & $0,360^{* *}$ \\
\hline
\end{tabular}

** Correlaciones Pearson con $p<, 01, *$ Correlaciones Pearson con $p<, 05$

\section{Discusión}

La evaluación de los estilos de afrontamiento en pacientes oncológicos es de relevancia debido a que representa una variable que puede coadyuvar a una mayor adaptación y solución de problemas ante la enfermedad y en sentido contrario una mayor disfunción psicológica. El instrumento de afrontamiento al cáncer derivado del $M A C^{(10)}$ en una muestra de pacientes con cáncer mexicanos ha mostrado adecuadas características psicométricas replicadas en estudios de diversos países.

De acuerdo a la presente investigación, el factor espíritu de lucha con un $\alpha=0,80$, concuerda con alfas de 0,54 a 0,89 reportadas de la versión original y en diferentes validaciones $^{(10,12,13,18,19)}$. De acuerdo a Sarason y Sarason ${ }^{(26)}$ los estilos de afrontamiento dirigidos al problema se relacionan con recursos personales que incluyen la capacidad para: buscar información pertinente; compartir preocupaciones y encontrar consuelo cuando se necesita; volver a definir una situación de manera que se pueda facilitar su solución; considerar las alternativas y analizar las consecuencias; emplear el sentido del humor para hacer menos tensa una situación.

En población oncológica ${ }^{(27)}$ mencionan que los estilos de afrontamiento centrados en el problema están dirigidos a manejar las demandas internas y externas que implican el diagnóstico, tratamiento, pronóstico y seguimiento de la enfermedad, caracterizada por una mejor adaptación al proceso oncológico. Esto concuerda con la literatura que identifica dichos estilos asociados con una mayor adherencia terapéutica, mejor adaptación ante la enfermedad y tratamiento, mayor calidad de vida, satisfacción con la vida y el futuro; así como percepción de autoeficacia principalmente en los estadios iníciales de la enfermedad ${ }^{(28-30)}$.

El factor Preocupación Ansiosa obtuvo un $\alpha=0,71$; otros estudios identifican alfas de 0,60 a 0,87 en este factor ${ }^{(10,12-14,19)}$, esto es relevante porque la ansiedad es particularmente alta al inicio del tratamiento oncológico particularmente al inicio de la quimioterapia ${ }^{(31,32)}$ por lo cual niveles altos de ansiedad pueden llegar a disminuir la adaptación del pacientes durante el proceso de atención a su salud. 
El factor Desamparo/Desesperanza, con un $\alpha=0,70$ que es menor en comparación con otros estudios (alfas de 0,75 a 0,91$)^{(12-15,18,19)}$ denominado sólo desesperanza. $\mathrm{La}$ desesperanza ante la enfermedad, hace referencia a la capitulación de los esfuerzos, ya que la persona siente que nada puede cambiar o hacer ${ }^{(33)}$. Este estilo puede afectar al desarrollo del cáncer de manera indirecta, por ejemplo, haciendo que los sujetos tarden más en buscar atención médica y menor adherencia a la medicación (34). Finalmente estos estilos de afrontamientos se asocian con menor calidad de vida y mayores niveles de ansiedad, depresión y dolor ${ }^{(3,35,36)}$.

En relación al factor actitud positiva, aunque este factor no se contempla en la versión original, ha sido identificado en otros estudios ${ }^{(14,17,19)}$. La actitud positiva se basa en un modelo de auto-regulación conductal, según el cual, un individuo persiste en conseguir un resultado si éste es percibido como alcanzable; es decir, la creencia general de que el futuro le va a deparar a uno más resultados positivos que negativos ${ }^{(37,38)}$. En población mexicana podría expresarse como lo señala Knaul( ${ }^{(39)}$ " $e l$ proceso de aprender cómo encontrar formas y fórmulas de vivir sin lamentar lo que perdi con el tratamiento. Hay que procesar las pérdidas y el duelo para no hundirse en ello. Visto de otra forma, de la forma correcta, es una oportunidad y un momento de redimensionar y ser creativo -oportunidades que pocas veces encuentra uno en la vida adulta por tener que enfrentar las presiones y el tren rutinario de la vida diaria."

Respecto a la evitación cognitiva representa el factor con la menor consistencia interna, este componente ha sido identificado en otros estudios con alfas de 0,65 a $0,82^{(13,17,19)}$. Se debe destacar que este factor en población mexicana parece no ser totalmente desadaptativa, pues muestra una asociación positiva significativa con el estilo de afrontamiento de espíritu de lucha.

La ausencia del factor fatalismo identificado en la versión original de Watson et al. ${ }^{(10)} \mathrm{y}$ en otros estudios ${ }^{(13,14,17,18)}$ puede explicarse por posibles características culturales de la muestra mexicana como el uso de la fe, esperanza y espiritualidad como estrategia para afrontar eventos estresantes, así como que algunos ítems fueron se integraron a los factores desesperanza y preocupación ansiosa. Esto podría indicar que la muestra mexicana emplea estrategias orientadas a la ansiedad y/o desesperanza, que conlleva una valoración del cáncer y su tratamiento como amenaza y poco control; sin llegar a presentar una actitud de aceptación frente al desenlace "resignándose a su suerte".

En cuanto a la varianza explicada, el presente estudio identifica un porcentaje promedio respecto a otros estudios $(36,67 \%-70 \%)^{(13-15,17,19)}$. Sobre la validez convergente se identificaron correlaciones bajas y medias negativas principalmente entre depresión-ansiedad y espíritu de lucha; y positivas con desamparo/desesperanza; llama la atención la asociación positiva significativa de la evitación con el estilo de afrontamiento de espíritu de lucha, lo cual indica que esta parece no ser tan disfuncional. La validez convergente concuerda con lo reportado en otros estudios ${ }^{(12,15,17,19,40)}$.

El actual estudio mostró un instrumento de 22 ítems, el cual se puede explicar adicionalmente por las diferencias clínicas identificadas en población mexicana como que el $23,3 \%$ presentaba una comorbilidad, lo cual implica que este grupo tiene que lidiar con dos o más enfermedades concurrente y probablemente con esquemas de tratamiento más complejos, así como que el $60,9 \%$ estaba solo en quimioterapia lo cual es diferente a muestras de otros estudios. Por otra parte las correlaciones entre factores se puede considerar que cada uno de estos representa respuestas relativamente independientes. En este punto hay que considerar que Watson et al. ${ }^{(10)}$ no plantea 
un modelo de estado o de rasgo en la evaluación del afrontamiento al cáncer; en cambio, utilizan un modelo pragmático que evalúa respuestas de un individuo en un momento dado de su enfermedad y es de esperar que sus respuestas sean altamente estables si se mantiene estable su situación (p. ej. pronóstico o tratamiento).

Esta aproximación es consistente con el cuerpo de la investigación en afrontamiento y adaptación al cáncer, que señala que el afrontamiento a la enfermedad puede ser un proceso dinámico, que cambia según las circunstancias y la valoración continua del individuo acerca de su expectativa de vida o del cumplimiento de sus metas ${ }^{(41)}$. Finalmente son de relevancia las relaciones con medidas de ansiedad, depresión y distrés, que se muestran consistentes con estudios realizados, pues estas variables constituyen criterios de ajuste al cáncer, y en general a la enfermedad ${ }^{(42)}$.

El presente instrumento se puede considerar como una escala con aceptables características psicométricas, se recomienda su empleo al ser un instrumento breve, fácil de aplicar y confiable para la práctica clínica y la investigación. Varias razones pueden explicar los resultados factoriales diversos como muestras no comparables, diferencias culturales en las formas de afrontar el cáncer, así como diferentes procedimientos de rotación factorial. Se requieren más estudios en otras poblaciones que confirmen estos hallazgos.

\section{Referencias bibliográficas}

1. Marrero RR, Carballeira M. Terapia de solución de problemas en enfermos de cáncer. Inter J Psychol Psychol Ther 2002; 2: 23-39.

2. Brennan J. Adjustment to cancer-coping or personal transition?. Psycho-Oncology 2001; 10: $1-18$

3. Nezu A. Terapia de solución de problemas para pacientes con cáncer. Revista Mexicana de Psicología 2001;18:185-92.

4. Lazarus R, Folkman S. Estrés y procesos cognitivos. Barcelona: Martínez Roca, 1991.

5. Castillo-López RL, Marván Garduño ML, Galindo Vázquez O, Landa-Ramírez E. Afrontamiento en pacientes con cáncer de mama: una revisión narrativa. Revista Latinoamericana de Medicina Conductual 2017; 8: 35-41.

6. Tobin DL, Holroyd KA, Reynolds RV, Kigal JK.m The hierarchical factor structure of the Coping Strategies Inventory. Cognit Ther Res 1989;13:343-61. doi:10.1007/ BF01173478.

7. Watson M, Greer S, Blake S, Shrapnell K. Reaction to a diagnosis of breast cancer relationship between denial, delay and rates of psychological morbidity. Cancer 1984;53: 2008-12. doi: 10.1002/1097-0142(19840501)53:93.0.CO;2-B

8. Moorey S, Frampton M, Greer S. The Cancer Coping Questionnaire: A self-rating scale for measuring the impact of adjuvant psychological therapy on coping behaviour. Psychooncology 12:331-44, 2003. doi: 10.1002/pon.646

9. Watson M, Homewood J. (2008). Mental Adjustment to Cancer ScaleO: psychometric properties in a large cancer cohort. Psychooncology 2008;17:1146-51. doi: 10.1002/ pon. 1345.

10. Watson M, Greer S, Young J, Inayat Q, Burgess C, Robertson C. Development of a questionnaire measure of adjustment to cancer: the MAC scale. Psychol Med 1988;18:2039, 1988. doi: 10.1017/S0033291700002026 
11. Watson M, Law MG, Santos MD, Greer S, Baruch J, Bliss J. The Mini-MAC: further development of the mental adjustment to cancer scale. J Psychosoc Oncol 1994;12:33-46. doi: 10.1300/J077V12N03_03

12. Cayrou S, Dickés P, Gauvain-Piquard A, Roge B. The Mental Adjustment to Cancer (MAC) Scale: French replicarion and assessment of positive and negative adjustment dimensions. Psychooncology 2003;12:8-23. doi: 10.1002/pon.634

13. Grassi L, Buda P, Cavana L, Annunziata MA, Torta R, Varetto A. Styles of coping with cancer: The Italian version of the Mini-Mental Adjustment to Cancer (Mini-MAC) scale. Psychooncology 14:115-24, 2005. doi: 10.1002/pon.826

14. Mystakidou K, Watson M, Tsilika E, Parpa E, Primikiri A, Katsouda E, Vlahos, L. Psychometric analyses of the Mental Adjustment to Cancer (MAC) scale in a Greek palliative care unit. Psychooncology 2005;14:16-24. doi: 10.1002/pon.801

15. Costa-Requena G, Gil F. The Mental Adjustment to Cancer Scale: A psychometric analysis in Spanish cancer patients. Psychooncology 2009;18:984-91. doi: 10.1002/pon.1466

16. Forero F, Bernal G, Restrepo M. Propiedades psicométricas de la Escala de Ajuste Mental al Cáncer (MAC) en una muestra de pacientes colombianos. Avances en Medición 2005;3:13552.

17. Ho SMY, Kam Fung W, Chan CLW, Watson M, Tsui YKY. Psychometric properties of the Chinese version of the Mini-Mental Adjustment to Cancer (MINI-MAC) scale. Psychooncology 2003;12:547-56. doi: 10.1002/pon.672

18. Akechi T, Fukue-Saeki M, Kugaya A, Okamura H, Nishiwaki Y, Yamawaki S, Uchitomi Y. Psychometric properties of the Japanese version of the mental adjustment to cancer (MAC) scale. Psychooncology 2000;9:395-401. 10.1002/1099-1611(200009/10)9:5<395::AIDPON472>3.0.CO;2-O

19. Kang JI, Chung HC, Kim SJ, Choi HJ, Ahn JB, Jeung HC, et al. Standardization of the Korean version of Mini-Mental Adjustment to Cancer (K-Mini-MAC) scale: factor structure, reliability and validity. Psychooncology 2008;17:592-7. doi: 10.1002/pon.1277

20. Swchartz CE, Daltroy LH, Brandt U, Friedman R, Stolbach L. A psychometric analysis of the Mental Adjustment to Cancer Scale. Psychol Med 1992; 22:203-10.

21. Nordin K, Berglund G, Terje I, Glimelius B. The Mental Adjustment to Cancer Scale A psychometric analysis and the concept of coping. Psychoncology 1999;8:250-9. doi: 10.1002/pon.1466

22. Zigmond AS, Snaith RP. The Hospital Anxiety and Depression Scale. Acta psychiatric Scand 1983;67:361-370. doi: 10.1111/j.1600-0447.1983.tb09716.x Cited by: 4215

23. Galindo O, Benjet C, Juárez F, Rojas E, Riveros A, Aguilar-Ponce JL et al. Propiedades psicométricas de la Escala Hospitalaria de Ansiedad y Depresión (HADS) para pacientes con cáncer en población mexicana. Salud Mental 2015; 4:253-8. doi: 10.17711/SM.01853325.2015 .035

24. Holland JC, Anderson B. NCCN Guidelines for Distress Management. JNCCN 2007; 5: 6698.

25. Almanza JJ, Rosario I, Pérez S. Traducción, adaptación y validación del termómetro de distrés en una muestra de pacientes mexicanos con cáncer. Rev Sanid Milit Mex 2008;62:209-17.

26. Sarason I, Sarason B. Psicopatología anormal: el problema de la conducta inadaptada, Pearson Ed, Mexico. 2006

27. Greer S, Moorey S, Barauch JD. Evaluation of adjuvant psychological therapy for clinically referred cancer patients. Br J Cancer 1991;63:257-60. 
28. Jim H, Richardson SA, Golden-Kreutz DM, Andersen BL. Strategies used in coping with a cancer diagnosis predict meaning in life for survivors. Health Psychol 2006; 25:753-61. doi: 10.1037/0278-6133.25.6.753

29. Roesch S, Weiner B. A meta-analytic review of coping with illness: Do causal attributions matter? J Psychosom Res 2001;41:813-19.

30. Taylor SE, Stanton AL. Coping resources, coping, processes, and mental health. Ann Rev Clin Psychol 2007;3:377-401. doi: 10.1146/annurev.clinpsy.3.022806.091520

31. Bronner MB, Nguyen MH, Smets EM, van de Ven AW, van Weert JC. Anxiety during cancer diagnosis: Examining the influence of monitoring coping style and treatment plan. Psychooncology 2018;27:661-7. doi: 10.1002/pon.4560

32. Lee MS, Tyson DM, Gonzalez BD, Small BJ, Lechner SC, Antoni M et al. Anxiety and depression in Spanish-speaking Latina cancer patients prior to starting chemotherapy. Psychooncology. 2018;27:333-8. doi: 10.1002/pon.4462

33. Várela B, Delgado L, Viera M, Galain A, Schwartzmann L. Afrontando la posibiidad de tener cáncer de mama. Ciencia y enfermería 2007;13:33-40.

34. Schou I, Ekeberg Ø, Ruland CM, Sandvik L, Kåresen R. Pessimism as a predictor of emotional morbidity one year following breast cancer surgery. Psychooncology. 2004;13:309-20. doi: 10.1002/pon.747

35. Deimling GT, Wagner LJ, Bowman KF, Sterns S, Kercher K, Kahana B. Coping among older-adult, long-term cancer survivors. Psychooncology 2006;15:143-59. doi: 10.1002/ pon.931

36. Pulgar MÁ, Garrido S, Muela JA, del Paso GAR. Validación de un inventario para la medida del estrés percibido y las estrategias de afrontamiento en enfermos de cáncer (ISEAC). Psicooncología 2009;6:167-90.

37. Raji MA, Ostir GV, Markides KS, Goodwin JS. The interaction of cognitive and emotional status on subsequent physical functioning in older Mexican Americans: findings from the Hispanic established population for the epidemiologic study of the elderly. J Gerontol A Biol Sci Med Sci 2002;57:678-82.

38. Carver CS, Smith RG, Antoni MH, Petronis VM, Weiss S. Optimistic personality and psychosocial well-being during treatment predict psychosocial well-being among long-term survivors of breast cancer. Health Psychol 2005;24:508-16. doi: 10.1037/0278-6133.24.5.508

39. Knaul FM. Una mujer ante el cáncer de mama en México. Salud pública de México. 2009;51:s364-s371.

40. Bredal IS. The Norwegian version of the Mini-Mental Adjustment to Cancer Scale: factor structure and psychometric properties. Psychooncology 2010;19:216-21. doi: 10.1002/ pon. 1564

41. Rowland J. Intrapersonal Resources: Coping. En Holland J, Rowland J, editors. Handbook of Psychooncology. Psychological care of the patient with cancer. New York: Oxford University Press 1990. p. 44-57

42. Livneh H, Antonak R. Psychosocial adaptation to chronic illness and disability: A primer for counselors. J Couns Develop 2005;83:12-20. doi: 10.1002/j.1556-6678.2005.tb00575.x 